\title{
One-Point Closure Models for Buoyancy-Driven Turbulent Flows
}

\author{
K. Hanjalić \\ Department of Applied Physics, Delft University of Technology Lorentzweg 1, \\ 2628 CJ Delft, The Netherlands; e-mail: hanjalic@ws.tn.tudelft.nl
}

\begin{abstract}
Key Words turbulence models, second-moment closure, buoyancy-driven flows, natural convection, RANS, T-RANS
\end{abstract}

- Abstract Turbulent flows driven by thermal buoyancy are featured by phenomena that pose a special challenge to conventional one-point closure models. Inherent unsteadiness, energy nonequilibrium, counter-gradient diffusion, strong pressure fluctuations, and lack of universal scaling, all believed to be associated with distinct large-scale coherent eddy structures, are hardly tractable by Reynolds-type averaging. Second-moment closures, though inadequate for providing information on eddy structure, offer better prospects than eddy-viscosity models for capturing at least some of the phenomena. For some configurations (e.g., with heating from below), unsteady computational solutions of ensemble-averaged equations, using a one-point closure as the subscale model, may be unavoidable for accurate prediction of flow details and wall heat transfer. This article reviews the rationale and some specific modeling issues related to buoyant flows within the realm of one-point closures. The inadequacy of isotropic eddy-diffusivity models is discussed first, followed by the rationale of the second-moment modeling and its term-by-term scrutiny based on direct numerical simulations (DNS). Algebraic models based on a rational truncation of the differential second-moment closure are proposed as the minimum closure level for complex flows. These closures are also recommended as subscale models for transient statistical modeling (T-RANS) and very large eddy simulations (VLES). Examples of applications illustrate some recent achievements.

\section{INTRODUCTION}

Turbulent flows driven or influenced by thermal buoyancy are frequently encountered in many technological applications, such as building structures, space heating and cooling, smoke and fire spreading, nuclear reactor containment, radioactive waste containers, electronics equipment, solar collectors, and crystal growth. Environmental flows in the atmosphere and water accumulations are also dominated by buoyancy force. For many cases of practical relevance there are still no reliable tools for predicting even the integral heat and mass transfer coefficients, whereas the estimate of flow details, local heat transfer, and scalar transport—often of crucial 
importance for evaluating equipment performance or operational safety-are beyond the reach of common engineering methods. Computational fluid dynamics (CFD) and computational heat and mass transfer (CHMT) with a turbulence closure model for either the full spectrum [Reynolds-averaged Navier-Stokes (RANS)] or for a part of it with full resolution of (very) large eddies (LES, VLES) are the only tools that can provide such information for complex flows.

One-point turbulence closure models for RANS equations are now the mainstay of the computational methods for industrial turbulent flows and convective heat and mass transfer. Despite many ad hoc assumptions and intuitive and heuristic rationale, these models have proved remarkably successful in a wide range of applications. The past two decades have witnessed extensive validation in an increasing variety of flows based on direct numerical simulations (DNS), which has enabled a better distinction between the potentials of various models. It is generally recognized that the two-equation $k-\varepsilon$ and similar eddy-viscosity models with linear stress-strain relations and their analogue for scalar fields cannot reproduce any flows with significant nonequilibrium effects, flows subjected to body forces or to any extra-strain rates other than simple shear. In flows driven purely by thermal buoyancy, the deficiencies of isotropic eddy-diffusivity models are even more transparent. Differential second-moment closures, or even their algebraic derivatives, offer a sounder physical basis for reproducing more accurately the turbulence dynamics and mean flow properties. A major advantage is in the possibility for exact treatment of some important turbulence interactions (e.g., stress and flux production, effects of rotation, buoyancy) or for a sounder modeling of the remaining terms by introducing stress and flux anisotropy parameters. However, higher-order models do not give a priori decisive advantages and more reliable predictions in every flow situation. For flows governed by thermal or concentration buoyancy, second-moment closures contain a large number of terms that need to be modeled and for which scarce experimental and DNS data are available. Because of a need to model more terms, the second-moment closures bring in more uncertainty and possibilities for ill-founded and unrealistic models of specific interactions, which can annul natural advantages of the method. DNS of some simple flows have revealed how poorly most current closures, including the second-moment ones, reproduce individual budget terms, even though they may predict mean flow properties well in accord with those obtained by experiments. Second-moment closures are also computationally more demanding. Because of these and other shortcomings, there is a view among the CFD community that second-moment closures have not fulfilled the early expectation in demonstrating indisputable superiority over simpler two-equation $k-\varepsilon$ and similar models.

There are also other features that make the RANS modeling of buoyancydriven flows exorbitant. Such is a departure from local energy equilibrium both in physical and spectral space. Buoyancy produces a unidirectional stratification and, depending on the orientation of the temperature-gradient vector imposed by the 
boundary conditions with respect to the gravitation vector, a variety of regimes may coexist. Even at relatively high bulk-Rayleigh numbers it is common to encounter regions with stagnant fluid, laminar circulation, the transitional regime, and fully turbulent regions-all in one flow. This emphasizes the role of molecular effects both close to a solid boundary and away from walls (at the edge of turbulent flows) and the need to use models that can universally account for low-Reynolds- and Péclet-number phenomena, irrespective of the wall vicinity. On the other hand, strong variation of all flow properties in usually very thin boundary layers along the walls, where the buoyancy exhibits the strongest effects on turbulence, requires a fine numerical resolution of the near-wall region and adequate modeling of both the viscous and conduction effects as well as nonviscous, nonconduction wallblockage effects. To this one may add a lack of universal scaling for different boundary conditions (orientation of heated walls), which hampers prospects for computational bridging of the near-wall regions with "wall functions" as practiced for forced flows.

Whereas some of these deficiencies can be cured or diminished by improved modeling, phenomena associated with well-organized coherent large-eddy structure, such as convective roll cells, plumes, and thermals, cannot be satisfactorily reproduced by traditional one-point closures irrespective of the closure level used. Such are the true or apparent counter-gradient diffusion (e.g., Kenjereš \& Hanjalić 1995, 1999a), augmented transport by pressure fluctuations (Wörner and Grözbach 1997), or strong variation of wall Nusselt numbers-especially noticeable in flows over horizontal heated walls. Because most one-point closures use only a single scalar turbulence time or length scale to characterize various interactions in turbulence dynamics, they cannot account for any spectral interactions, provide structural information, or detect any identifiable eddy structure. Some onepoint closures partially compensate for these deficiencies by various additions to the basic model to account indirectly for spectral nonequilibrium. Most of these remedies have limited applications and usually fail when the flow is governed by dominant and well-organized large-scale structures. Capturing these structures can only be done by performing time-dependent simulation, such as DNS or LES. Because of the need to also resolve the thin wall boundary layer and the wall heat transfer, their application is limited to relatively low Rayleigh and Reynolds numbers (Grözbach 1982, Kerr 1996). A middle of the road solution is to resolve in time and space only very large coherent eddy structure (very large eddy simulation) while modeling the "rest" of the turbulence with a one-point closure model. Such an approach, termed T-RANS (time-dependent RANS), which uses a conventional algebraic stress/flux closure as the "subscale" model, has proved successful particularly in predicting buoyant flows heated from below (Hanjalić \& Kenjereš 2000b).

This article begins with a DNS-based term-by-term scrutiny of models of various terms in the transport equation for the turbulent heat flux in the traditional RANS models for buoyancy-driven flows. Obvious deficiencies are identified and 
some new proposals for model improvement are presented, illustrated by a priori validation in two generic cases, one with heating from sides and one from below. DNS results are also used to validate the common differential-to-algebraic truncation of the scalar flux equation. Despite the deficiency in this assumption, a $k-\varepsilon-\overline{\theta^{2}}$ algebraic model reproduces well the mean flow properties, turbulence second moments, and wall heat transfer in a variety of wall-bounded buoyant flows.

The article closes with a brief discussion of the T-RANS approach in which the large-scale deterministic structure is fully resolved, whereas the stochastic turbulence is modeled by the same algebraic one-point closure. It is shown that even the two-dimensional T-RANS can capture the ensemble-averaged convective roll cell patterns and wall heat transfer distribution in Rayleigh-Bénard convection and similar flows with one homogeneous direction (Kenjereš \& Hanjalić 2000). Finally, the potential of the three-dimensional T-RANS simulation is illustrated in the example of a Rayleigh-Bénard convection at very large Rayleigh numbers, up to $10^{15}$, which are at present inaccessible by LES.

\section{ONE-POINT RANS CLOSURES FOR BUOYANT FLOWS: CLOSURE LEVELS AND THEIR LIMITATIONS}

\subsection{Eddy-diffusivity Models}

The Reynolds-averaged momentum and energy equations governing turbulent flows subjected to body forces are

$$
\begin{array}{ll}
\frac{\mathrm{D} U_{i}}{\mathrm{D} t} & =F_{i}-\frac{1}{\rho} \frac{\partial P}{\partial x_{i}}+\frac{\partial}{\partial x_{j}}\left(v \frac{\partial U_{i}}{\partial x_{j}}-\overline{u_{i} u_{j}}\right), \quad \text { and } \\
\frac{\mathrm{D} T}{\mathrm{D} t} & =\frac{q}{\rho c_{p}}+\frac{\partial}{\partial x_{j}}\left(\frac{v}{\sigma_{T}} \frac{\partial T}{\partial x_{j}}-\overline{\theta u_{j}}\right),
\end{array}
$$

where $\mathrm{D} / \mathrm{D} t=\partial / \partial t+U_{k} \partial / \partial x_{k}$ is the material derivative, $F_{i}$ is the body force acting on the fluid (thermal and concentration buoyancy, Lorentz or Corriolis force), and $q$ is the internal energy sources. An analogous equation can be written for species concentration.

To close the equations, the most frequent approach is to use simple eddyviscosity/diffusivity models for turbulent flux of momentum $\overline{u_{i} u_{j}}$ and of heat $\overline{\theta u_{j}}$ (or species):

$$
\overline{u_{i} u_{j}}=\frac{2}{3} k \delta_{i j}-v_{t}\left(\frac{\partial U_{i}}{\partial x_{j}}+\frac{\partial U_{j}}{\partial x_{i}}\right) \quad \text { and } \quad \overline{\theta u_{i}}=-\frac{v_{t}}{\sigma_{T}^{t}} \frac{\partial T}{\partial x_{i}},
$$

where $k=\overline{u_{i} u_{j}}$ is the turbulence kinetic energy, $v_{t}$ is the eddy viscosity, usually defined as $C_{\mu} k^{2} / \varepsilon$, and $\varepsilon$ is the kinetic energy dissipation rate obtained from a separate transport equation. 
The first obvious deficiency of Equations 3 for flows driven by thermal buoyancy and other body forces is the absence of body-force source terms $\overline{f_{i} u_{j}}+\overline{f_{j} u_{i}}$ and $\overline{f_{j} \theta}$, which appear in the full transport equations for $\overline{u_{i} u_{j}}$ and $\overline{\theta u_{j}}$, respectively. Next, the expression for heat flux vector $\overline{\theta u_{i}}$ implies that its components are aligned with the corresponding components of the temperature-gradient vector, with an isotropic eddy diffusivity as the proportionality factor. The alignment of the heat flux with the mean temperature-gradient vector leads to model failure in many cases, with the two canonic situations being $(a)$ a fluid layer heated from below (Rayleigh-Bénard convection or a penetrative convective layer) and (b) the natural convection along a heated vertical wall (Figures $1 a$ and $1 b$ ). In the first case, which in the long-term average is horizontally homogeneous, the mean temperature becomes almost uniform owing to extensive vertical mixing (except for a thin conduction layer near the wall). The only nonzero component of heat flux in the vertical direction has, therefore, no relation with the almostzero (or even slightly positive) temperature gradient in the vertical direction, as implied by the isotropic eddy-diffusivity model. The same problem is encountered in double diffusive systems with thermal and concentration buoyancy: in mixed layers dominated by unstable thermal stratification, both the long-term averaged temperature and concentration are uniform. In a convective boundary layer along a heated vertical wall, the major buoyancy source of turbulence kinetic energy is associated with the vertical heat flux $\beta g_{i} \overline{\theta u_{i}}$, where $\beta$ is the thermal expansion coefficient $-1 / \rho(\partial \rho / \partial T)_{C, P}$. Associating the heat flux $\overline{\theta u_{i}}$ with the usually negligible (zero for an infinite plate) mean temperature gradient in the vertical direction will eliminate the buoyancy effect on turbulence, contrary to the basic physics of the buoyancy-driven turbulence.

Further illustrations are provided in Figures $2 a$ and $2 b$. The isotropic eddy diffusivity is usually related to the eddy viscosity $v_{t}$ via the turbulent Prandtl
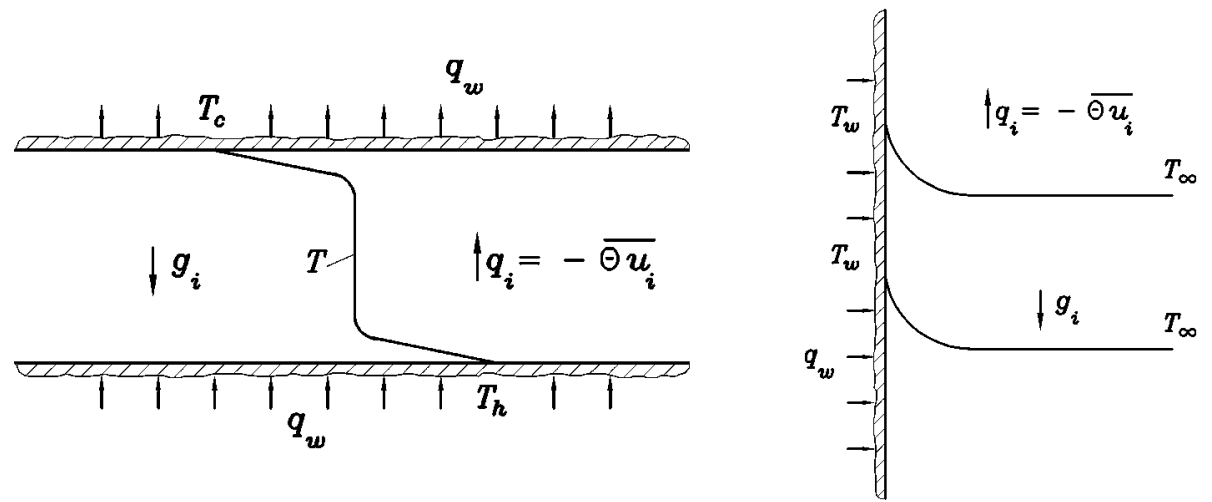

Figure 1 Sketch of Rayleigh-Bénard convection, $\nabla T \| \vec{g}$, (left) and of a vertical heated wall, $\nabla T \perp \vec{g}$, (right). 
(b)
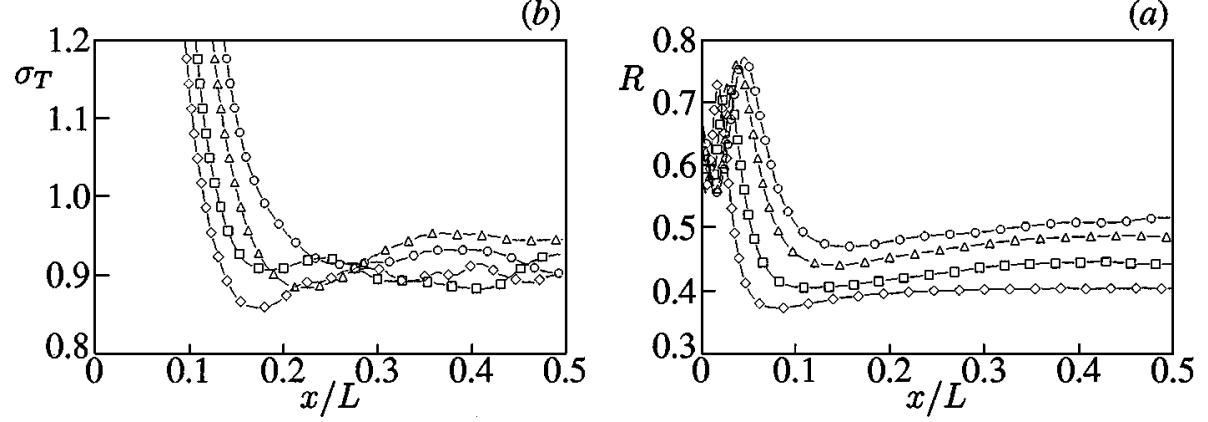

Figure 2 The timescale ratio $R(a)$ and the turbulent Prandtl number $\sigma_{T}(b)$ in a vertical side-heated channel at several Rayleigh numbers (-o-, $R a=5.4 \times 10^{5} ;-\triangle-, R a=8.227 \times$ $\left.10^{5} ;-\square-, R a=2 \times 10^{6} ;-\diamond-, R a=5 \times 10^{6}\right)$ (Dol et al. 1999).

number $\sigma_{T}^{t}$, which is assumed constant $(\approx 0.9)$. Inadequacy of such an assumption is illustrated in Figure $2 a$, which shows the DNS variation of $\sigma_{T}^{t}=$ $\left(\overline{u_{1} u_{2}} d T / d x_{1}\right) /\left(\overline{u_{1} \theta} d U_{2} / d x_{1}\right)\left(x_{1} \equiv x\right.$ is horizontal direction) with wall distance and Rayleigh numbers in an infinite, side-heated vertical plane channel (Dol et al. 1999, Versteegh 1998). Another frequent assumption (also used in secondmoment closures) concerns the mechanical and thermal timescales, $\tau=k / \varepsilon$ and $\tau_{t h}=\overline{\theta^{2}} / \varepsilon_{\theta \theta}$, respectively, where $\overline{\theta^{2}}$ is the temperature variance and $\varepsilon_{\theta \theta}$ its dissipation rate. Whereas a model transport equation for $\overline{\theta^{2}}$ can be easily derived and solved, this is not the case for $\varepsilon_{\theta \theta}$. The latter is then usually provided by assuming that the mechanical and thermal timescales are proportional to each other, i.e., $R=\tau_{\theta} / \tau \approx 0.5$. An example of the inadequacy of such an assumption (which essentially implies the use of a single scale to model both the mechanical and thermal turbulence) is illustrated in Figure $2 b$, obtained from DNS of flow in a side-heated vertical plane channel) (Dol et al. 1999) (discussed below).

\subsection{Second-Moment Closures}

Some of the above shortcomings can be eliminated by solving the modeled transport equations for $\overline{u_{i} u_{j}}$ and $\overline{\theta u_{j}}$, closed by the equations that provide turbulence mechanical and thermal scales (e.g., for $\overline{\theta^{2}}, \varepsilon$, and $\varepsilon_{\theta \theta}$ ). Such complete secondmoment closures, involving 17 differential transport equations, have been proposed in the literature and applied with success to the computations of some simple buoyancy-driven flows. However, such models contain many terms that require separate modeling, and deriving a general closure for complex flows is a formidable task. Considering modeling issues in all equations is beyond the scope of this article, and we focus only on the transport equations for the turbulent scalar flux and temperature variance, which are the major modeling targets in buoyant 
convection. The exact heat flux equation can be written as

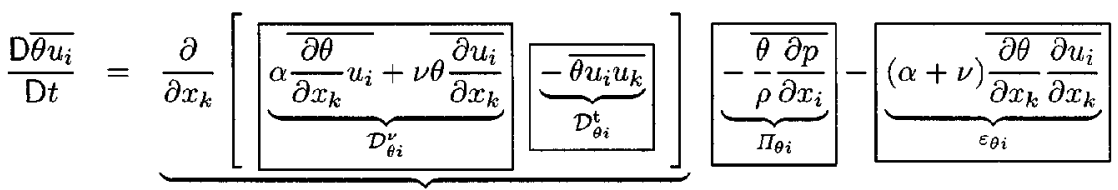

$$
\begin{aligned}
& \underbrace{\underbrace{-\overline{u_{i} u_{k}} \frac{\partial T}{\partial x_{k}}}_{P_{\theta i}^{T}} \underbrace{-\overline{\theta u_{k}} \frac{\partial U_{i}}{\partial x_{k}}}_{P_{\theta i}^{U}} \underbrace{-g_{i} \beta \overline{\theta^{2}}}_{G_{\theta i}^{t h}}+\underbrace{g_{i} \beta_{c} \overline{\theta c}}_{G_{\theta i}^{c}}+\underbrace{\overline{f_{i}^{L} \theta}}_{G_{\theta i}^{L}}+\underbrace{\overline{f_{i}^{r} \theta}}_{G_{\theta i}^{R}}}_{\text {Production }},
\end{aligned}
$$

where $\mathcal{D}_{\theta i}$ stands for the total diffusion of $\overline{\theta u_{i}}$ (molecular $\mathcal{D}_{\theta i}^{v}$ and turbulent $\mathcal{D}_{\theta i}^{t}$ ), $\Pi_{\theta i}$ is the pressure scrambling effect, $\varepsilon_{\theta i}$ is the molecular destruction, and the rest are the production terms. In addition to the production due to mean temperature and velocity gradients, $P_{\theta i}^{T}$ and $P_{\theta i}^{U}$, respectively, the equation contains production due to body forces: thermal buoyancy $G_{\theta i}^{t h}$, concentration buoyancy $G_{\theta i}^{c}$ [where $\beta_{c}=1 / \rho(\partial \rho / \partial C)_{T, P}$ is the volume expansion coefficient due to concentration stratification], magnetic field (Lorentz force) $G_{\theta i}^{L}$, and system rotation $G_{\theta i}^{\Omega}$ (the latter three are not considered further).

It is noted that terms in boxes, $\mathcal{D}_{\theta i}, \Pi_{\theta i}$ and $\varepsilon_{\theta i}$, need to be modeled (including molecular diffusion, if $P r \neq 1$ ), whereas other terms can be treated in an exact manner, in the sense that variables in these terms are provided from their own, though modeled, transport equations. For example, for production due to thermal buoyancy it is essential to consider the transport equation for the temperature variance

$$
\frac{\mathrm{D} \overline{\theta^{2}}}{\mathrm{D} t}=\underbrace{\frac{\partial}{\partial x_{k}}[\underbrace{\alpha \frac{\partial \overline{\theta^{2}}}{\partial x_{k}}}_{\mathcal{D}_{\theta \theta}^{\nu}}[\underbrace{-\overline{\theta^{2} u_{k}}}_{\mathcal{D}_{\theta \theta}^{\mathrm{t}}}]}_{\mathcal{D}_{\theta \theta}} \underbrace{-2 \overline{u_{k}} \frac{\partial T}{\partial x_{k}}}_{P_{\theta \theta}}-\underbrace{2 \alpha \frac{\partial \overline{\partial \theta} \frac{\partial \theta}{\partial x_{k}}}{\partial x_{k}}}_{\varepsilon_{\theta \theta}} .
$$

Models of various terms in Equations 4 and 5 have been proposed, primarily for forced convection (Launder 1976, Gibson \& Launder 1978, Shih et al. 1988) but also modified for and applied to buoyancy-driven flows (Peeters \& Henkes 1992, Dol \& Hanjalić 2001). However, it was only recently when DNS became available for some simple flows that a term-by-term validation and further model modification became possible. Yet this is not an easy task, even when confining attention to only simple canonic flows. In the following section we discuss the common practice and some new developments in term-by-term modeling of Equations 4 and 5.

WALL LIMITS A solid wall affects the turbulence both by viscous and nonviscous suppression of turbulence fluctuations. Whereas the viscous effect is of a 
scalar nature, the nonviscous effect due to blockage (impermeability, pressure reflection) is dependent on the wall proximity and configuration. In the flow over heated walls the near-wall turbulence is further affected because the flow is driven by the imposed wall conditions. As no universal scaling of flow properties has been established for any class of flows driven by thermal buoyancy, prospects for deriving wall functions for bridging the viscous and conductive layers are slim. Consequently, the equations have to be integrated up to the wall with appropriate low turbulent Reynolds number and nonviscous modifications. Modeling the wall effects is one of the prime difficulties, but it is a crucial prerequisite for the simulation of flow properties near walls, especially for predicting wall friction and heat transfer.

Satisfying the limiting behavior of the models at the wall is one of the basic requirements of near-wall modeling. Expressions for the wall limits of all budget terms can be derived by using Taylor-series expansion for the flow variables in terms of only wall-normal coordinate, here denoted by $x$, (Dol et al. 1999):

$$
\begin{aligned}
& p=a_{p}+b_{p} x+c_{p} x^{2}+d_{p} x^{3}+\cdots \\
& u_{i}=a_{i}+b_{i} x+c_{i} x^{2}+d_{i} x^{3}+\cdots \\
& \theta=a_{\theta}+b_{\theta} x+c_{\theta} x^{2}+d_{\theta} x^{3}+\cdots,
\end{aligned}
$$

with $a_{i}=b_{1}=a_{\theta}=0$ (no-slip condition, continuity, and constant wall temperature). Based on this expansion, the budget terms for $\overline{\theta u_{i}}$ and $\overline{\theta^{2}}$ can be obtained. For example, the near-wall values for the horizontal ("1") and vertical ("2") flux component and temperature variance in a vertical channel are summarized in Table 1.

The budget terms that are at least of second order in $x$ are omitted as their wall limits are considered to be less important. For illustration and further discussion the wall limits are also shown for $\Phi_{\theta i}=\overline{\left(\partial \theta / \partial x_{i}\right) p} / \rho$ and $\mathcal{D}_{\theta i}^{p}=-\left(\partial \overline{\theta p} / \partial x_{i}\right) / \rho$, the sum of which yields the total pressure scrambling $\Pi_{\theta i}$.

The unknown correlations that appear in Table 1 can be extracted from the DNS data. The correlations $\overline{b_{\theta} a_{p}} / \rho, \overline{b_{\theta} b_{2}}$, and $\overline{b_{\theta}^{2}}$ are directly available through the wall values of the budget terms in which they appear. The other correlations have to be determined from the near-wall slopes of the corresponding budget terms. The analysis of Dol et al. (1999) for the vertical channel, based on DNS data for several $R a$ numbers between $5.4 \times 10^{5}$ to $5 \times 10^{6}$, showed a slight $R a$-number dependence, probably due to relatively low $R a$ numbers, as also depicted in Figures $2 a$ and $2 b$.

Rayleigh- and Reynolds-number independence is an important criterion for judging the model generality. Dol et al. (1999) showed that the model they proposed (discussed below) performs almost equally well over one decade of Rayleigh numbers (the range for which the DNS data are available). A proof of the $R a$ number independence can be displayed only if proper scaling is applied separately 
TABLE 1 Near-wall behavior of viscous diffusion, pressure terms, and dissipation (leading terms of their Taylor-series expansions)

\begin{tabular}{|c|c|c|c|}
\hline$i$ & 1 & 2 & $\theta$ \\
\hline $\mathcal{D}_{\theta i}^{\nu}$ & $2(\alpha+2 \nu) \overline{b_{\theta} c_{1}} x$ & $(\alpha+\nu) \overline{b_{\theta} b_{2}}$ & $2 \alpha \overline{b_{\theta}^{2}}$ \\
\hline$\hat{\mathcal{D}}_{\theta i}^{\nu}$ & $3(\alpha+\nu) \overline{b_{\theta} c_{1}} x$ & $(\alpha+\nu) \overline{b_{\theta} b_{2}}$ & \\
\hline $\mathcal{D}_{\theta i}^{p}$ & $-\frac{1}{\rho} \overline{b_{\theta} a_{p}}-\frac{2}{\rho}\left(\overline{b_{\theta} b_{p}}+\overline{c_{\theta} a_{p}}\right) x$ & & \\
\hline$\Phi_{\theta i}$ & $\frac{1}{\rho} \overline{b_{\theta} a_{p}}+\frac{1}{\rho}\left(\overline{b_{\theta} b_{p}}+2 \overline{c_{\theta} a_{p}}\right) x$ & $\frac{1}{\rho} \overline{\left(\partial b_{\theta} / \partial y\right) a_{p}} x$ & \\
\hline$\Pi_{\theta i}$ & $-\frac{1}{\rho} \overline{b_{\theta} b_{p}} x$ & $\frac{1}{\rho} \overline{\left(\partial b_{\theta} / \partial y\right) a_{p}} x$ & \\
\hline$\varepsilon_{\theta i}$ & $2(\alpha+\nu) \overline{b_{\theta} c_{1}} x$ & $(\alpha+\nu) \overline{b_{\theta} b_{2}}$ & $2 \alpha \overline{b_{\theta}^{2}}$ \\
\hline
\end{tabular}

Note: terms in boxes are in balance at the wall.

for the near wall and in the central region of the channel. Dol et al. (1999) provided such a scaling, at least for the $R a$ range considered.

DIFFUSION In contrast to the Reynolds-stress and temperature-variance transport equations, molecular diffusive transport of $\overline{\theta u_{i}}$ has to be modeled if $\operatorname{Pr} \neq 1$. A model (marked with "hat") that satisfies the near-wall balance and the wall values (see Table 1) is given by the first term (between equal signs) in the following equation:

$$
\hat{\mathcal{D}}_{\theta i}^{v}=\frac{1}{2}(\alpha+v) \frac{\partial^{2} \overline{\theta u_{i}}}{\partial x_{k}^{2}}=\mathcal{D}_{\theta i}^{v}+\frac{1}{2}(\alpha-v) \overline{\frac{\partial^{2} u_{i}}{\partial x_{k}^{2}}}-\frac{1}{2}(\alpha-v) \frac{\overline{\partial^{2} \theta}}{\partial x_{k}^{2}} u_{i} .
$$

For $\operatorname{Pr}=1$ this term equals the exact diffusion $\mathcal{D}_{\theta i}^{v}$. If $\operatorname{Pr}$ differs very much from 1 , the remaining terms need to be modeled. However, in view of a large difference between the typical scales of the fluctuations and their second derivatives, the correlations in the last two terms of Equation 9 are small and for most cases of interest they can be neglected. Such a simplified expression shows excellent agreement with the DNS data of Versteegh (1998).

The turbulent-diffusion term in the heat flux equation contains the triple correlation $\overline{\theta u_{i} u_{k}}$. The usual modeling strategy is to simplify the exact transport equation for the triple correlation to an algebraic expression in terms of known quantities. Adopting a linear model of the "slow" pressure scrambling term (see below) and expressing fourth-order cumulants in terms of second moments while neglecting 
all transport terms leads to a set of closed, but interconnected, algebraic expressions for the triple moments:

$$
\begin{aligned}
\overline{u_{i} u_{j} u_{k}} & =-C \tau\left[\overline{u_{k} u_{l}} \frac{\partial \overline{u_{i} u_{j}}}{\partial x_{l}}+\cdots-C_{1} \beta\left(g_{k} \overline{\theta u_{i} u_{j}}+\cdots\right)\right] \\
\overline{\theta u_{i} u_{j}} & =-C \tau\left[\overline{u_{j} u_{k}} \frac{\partial \overline{\theta u_{i}}}{\partial x_{k}}+\cdots-C_{1} \beta\left(g_{i} \overline{\theta^{2} u_{j}}+\cdots\right)\right] \\
\overline{\theta^{2} u_{j}} & =-C \tau\left[\overline{u_{j} u_{k}} \frac{\partial \overline{\theta^{2}}}{\partial x_{k}}+\cdots-C_{1} \beta g_{j} \overline{\theta^{3}}\right] \\
\overline{\theta^{3}} & =-C \tau \overline{\theta u_{k}} \frac{\partial \overline{\theta^{2}}}{\partial x_{k}},
\end{aligned}
$$

where $C$ and $C_{1}$ stand for free coefficients to be determined (presumably different for each of the above expressions). Including all terms leads to a cumbersome model of triple moments (with many coefficients), which exceeds the level of approximation usually adopted for other terms in transport equation. Substantial simplification can be achieved by neglecting the buoyant terms in the above expression. For the heat flux equation this leads to the following model of turbulent diffusion:

$$
\hat{\mathcal{D}}_{\theta i}^{\mathrm{t}}=\frac{\partial}{\partial x_{k}}\left[C_{\theta} \frac{k}{\varepsilon}\left(\overline{u_{k} u_{l}} \frac{\partial \overline{\theta u_{i}}}{\partial x_{l}}+\overline{u_{i} u_{l}} \frac{\partial \overline{\theta u_{k}}}{\partial x_{l}}+\overline{\theta u_{l}} \frac{\partial \overline{u_{i} u_{k}}}{\partial x_{l}}\right)\right],
$$

usually with $C_{\theta}=0.11$. The last term of Equation 14, which has a character of an additional source in the equation for $\overline{\theta u_{i}}$, is often omitted, providing a simpler expression that consists of heat-flux gradients only and that is still invariant under coordinate rotation. Dol et al. (1997) showed that this simplification is useful and appropriate for the vertical channel. If the second term is also omitted, the wellknown gradient-diffusion model is obtained. That model, which is not invariant and performs inferiorly to the former one (see Dol et al. 1997), needs a larger coefficient, such as 0.22 .

A model for turbulent diffusion of the temperature variance can be derived in the same way. In this case, however, the production of the triple correlation $\overline{\theta^{2} u_{k}}$ needs to be included for acceptable performance (Dol et al. 1997). The invariant model expression is

$$
\begin{aligned}
\hat{\mathcal{D}}_{\theta \theta}^{\mathrm{t}} & =\frac{\partial}{\partial x_{k}}\left[C_{\theta \theta} \frac{k}{\varepsilon}\left(\overline{u_{k} u_{l}} \frac{\partial \overline{\theta^{2}}}{\partial x_{l}}+2 \overline{\theta u_{l}} \frac{\partial \overline{\theta u_{k}}}{\partial x_{l}}+2 \widehat{\theta u_{k} u_{l}} \frac{\partial T}{\partial x_{l}}\right)\right] \\
\widehat{\theta u_{i} u_{k}} & =-C_{\theta} \frac{k}{\varepsilon}\left(\overline{u_{k} u_{l}} \frac{\partial \overline{\theta u_{i}}}{\partial x_{l}}+\overline{u_{i} u_{l}} \frac{\partial \overline{\theta u_{k}}}{\partial x_{l}}\right) .
\end{aligned}
$$

Only thermal production is included because for an infinite vertical channel this is the only nonzero production budget term of $\overline{\theta^{2} u}$. The full expression needs 
$C_{\theta \theta}=0.05$. Omitting the last term of Equation 15 leads to the usual invariant model, with $C_{\theta \theta}=0.11$. The gradient-diffusion model, with $C_{\theta \theta}=0.22$, is obtained when only the first term is retained.

Although the above diffusion models reproduce well the DNS data for a vertical side-heated channel (see Dol et al. 1997), it should be noted that they do not work well in cases with heating from below, where the transport is dominated by organized convective roll cell structures. Some improvements can be achieved by more elaborate modeling of triple moments by resorting to the transport equations for triple moment and including terms other than the gradients of the second moments. This, however, does not work for moments involving fluctuating pressure. Wörner \& Grözbach (1997) found from DNS of Rayleigh-Bénard convection that the dominant turbulent transport of the kinetic energy in the vertical direction is by pressure fluctuations $\left(\overline{p u_{3}}\right)$ and not by velocity fluctuations $\left(\overline{k u_{3}}\right)$. Furthermore, the pressure diffusion was found to have an opposite sign so that the total turbulent transport occurs up the gradient of kinetic energy, contrary to the basic assumption of the gradient diffusion hypothesis proposed by Lumley (1978) (for more details, see also Hanjalić 1994). A way to avoid the problem of modeling the pressure diffusion is to retain and model the pressure scrambling term $\Pi_{\theta i}$ in its original form, as appears in Equation 4 instead of splitting it into "pressure-redistribution" and pressure diffusion (see below).

However, it should be noted that the prospect for capturing large-scale transport by any local gradient hypothesis is slim, and in situations where the large-scale coherent structure is dominant, this can only be successful with a full resolution of these structures as with LES or VLES (T-RANS).

DISSIPATION The dissipation rate of the turbulent heat flux is usually neglected. Although this is justified only when the turbulence is isotropic, at least at the smallest scales, in the flow in a vertical channel considered here, the DNS show that this is indeed a relatively small budget term, even near the walls where the largest and smallest scales are comparable. Unlike for the Reynolds stress, the dissipation does not balance the production. Instead, the production of $\overline{\theta u_{i}}$ is balanced mainly by the (total) pressure scrambling, which is a negative budget term throughout the channel. The dissipation of $\overline{\theta u_{i}}$ merely balances viscous diffusion in the near-wall region. Dol et al. (1999) proposed the following dissipation model that satisfies the above condition near a solid wall:

$$
\begin{aligned}
-\hat{\varepsilon}_{\theta i} & =-f^{*} \varepsilon_{\theta i}^{*}-\varepsilon_{\theta i}^{\prime} \\
-\varepsilon_{\theta i}^{*} & =-\frac{1}{2}\left(1+\frac{1}{\operatorname{Pr}}\right) \frac{\varepsilon}{k} \overline{\theta u_{i}} \\
-\varepsilon_{\theta i}^{\prime} & =-\frac{1}{2} \hat{\mathcal{D}}_{\theta i}^{v}+\frac{1}{4}\left(1+\frac{1}{\operatorname{Pr}}\right) \frac{\mathcal{D}_{k}^{v}}{k} \overline{\theta u_{i}} \\
f^{*} & =\exp \left(-\frac{3}{4} A^{3 / 2}\right),
\end{aligned}
$$


(a)

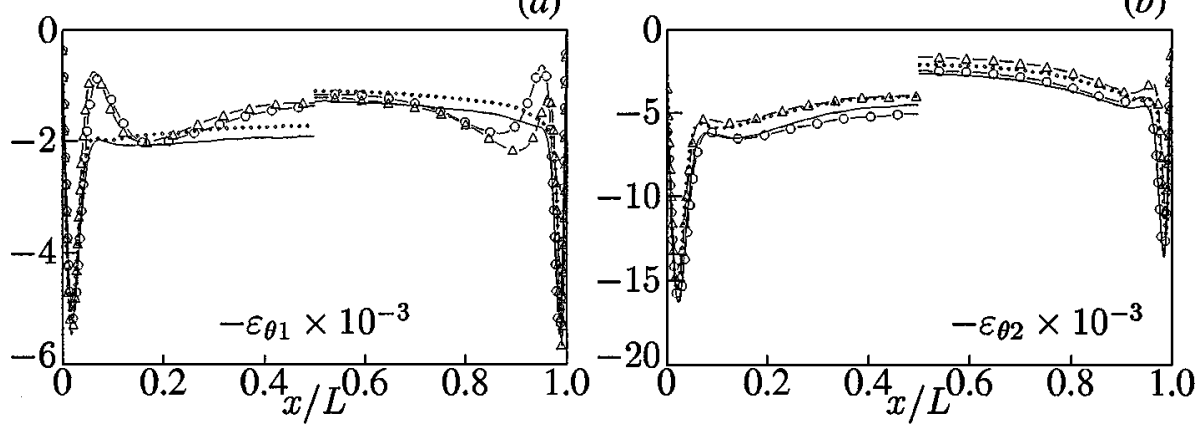

Figure 3 The new dissipation model (lines) compared with DNS (symbols) at several Rayleigh numbers. For $x / L<0.5$ :- and $-\circ-, R a=5.4 \times 10^{5} ; \cdots$ and $-\triangle-, R a=8.227 \times 10^{5}$. For $x / L>0.5$ : - and $-\circ-, R a=2 \times 10^{6} ; \cdots$ and $-\triangle-, R a=5 \times 10^{6}$ (Dol et al. 1999).

where $A$ is a stress invariant (see next paragraph). This model is obtained following that for the dissipation of the Reynolds-stress tensor of Hanjalić et al. (1997). The above model satisfies wall constraints using only local flow properties and is free from topological parameters such as wall distance or unit wall-normal vector. In Figure 3, Equation 17 is evaluated by feeding the DNS results for variables on the right-hand side of Equations 17-20 and comparing them with the DNS data for $\varepsilon_{\theta i}$. The figure shows that the new dissipation model performs equally well at all available Rayleigh numbers. Note that $\varepsilon_{\theta 1} \ll \varepsilon_{\theta 2}$, hence a finer scale is used in Figure $3 a$ so that a failure to reproduce the near-wall peak in $\varepsilon_{\theta 1}$ is not a serious deficiency of the model as a whole.

In the transport equation for the temperature variance, next to turbulent diffusion, only the dissipation needs modeling. Contrary to common belief, the dissipation rate $\varepsilon_{\theta \theta}$ is not the weak spot of the closure. For a vertical channel, as well as for several other flows tested, (see Section 3), assuming a constant thermal-tomechanical timescale ratio $R$ appears to be sufficient. The model then becomes $\hat{\varepsilon}_{\theta \theta}=\varepsilon \overline{\theta^{2}} /(R k)$. From Figure $2 a$, it follows that $R=0.5$ is a good choice for $0.1<x / L<0.5$ at $R a=5.4 \times 10^{5}$. Close to a wall for $x / L<0.1, R$ varies substantially exhibiting a peak around $x / L \approx 0.05$, but this behavior has a much smaller effect on $\hat{\varepsilon}_{\theta \theta}$ than expected (see Section 3). A Rayleigh-number dependence can be introduced: Dol et al. (1999) proposed $R=\min \left(2.2 R a_{\mathrm{t}}^{-0.13}, 0.75\right)$, which follows the DNS value for $R$ at $x / L=0.5$ accurately and which is almost constant for $0.1<x / L<0.5$ in the considered range of Rayleigh numbers. In this expression, $R a_{\mathrm{t}}=g \beta\left(\overline{\theta^{2}}\right)^{1 / 2} k^{9 / 2} \mathrm{Pr} /\left(v^{2} \varepsilon^{3}\right)$ is the turbulence Rayleigh number.

PRESSURE SCRAMBLING A common approach to modeling $\Pi_{\theta i}$ is to decompose it in several contributions corresponding to terms that appear in the exact Poisson integral for $\Pi_{\theta i}$, i.e., $\Pi_{\theta i}=\Pi_{\theta i, 1}+\Pi_{\theta i, 2}^{U}+\Pi_{\theta i, 2}^{T}+\Pi_{\theta i, 2}^{g}+\Pi_{\theta i}^{w}$, where index 1 
denotes the slow term (return to isotropy of anistropic turbulence in the absence of generation terms), 2 denotes the rapid terms (effect of mean flow deformation and body force), and superscript $w$ denotes wall effects. It is noted that the splitting of $\Pi_{\theta i}$ into redistribution and pressure diffusion, $\Pi_{\theta i}=\Phi_{\theta i}+\mathcal{D}_{\theta i}^{p}$, as practiced in modeling the stress equation, offers no advantage because $\Phi_{\theta i}$ is not redistributive and both terms are nonzero at the wall balancing each other. In buoyancydriven flows the pressure diffusion can be very large and even of the opposite sign from the velocity diffusion, thus being inconsistent with the local gradient hypotheses.

Various models for each part of $\Pi_{\theta i}$ (in fact, of $\Phi_{\theta i}$ ) have been proposed in the literature. The simplest first-order approximations, which reflect basic physics of the pressure-scrambling term, i.e., the isotropization of both the turbulence field and of its production (Launder 1976) are

$$
\begin{array}{ll}
\Pi_{\theta i, 1}=-C_{\theta 1} \overline{\theta u_{i}} / \tau & \Pi_{\theta i, 2}^{U}=-C_{\theta 2} P_{\theta i}^{U} \\
\Pi_{\theta i, 2}^{T}=-C_{\theta 2}^{\prime} P_{\theta i}^{T} & \Pi_{\theta i, 2}^{g}=-C_{\theta 3} G_{\theta i} .
\end{array}
$$

In the first approximation, the coefficients have been determined from experiments and numerical validation: $C_{\theta 1} \approx 3.0, C_{\theta 2}^{\prime}=0, C_{\theta 2}=C_{\theta 3} \approx 0.5 . \tau$ is the characteristic turbulence timescale, for which usually the mechanical scale is adopted, $\tau=k / \varepsilon$. It can be argued that a thermal timescale $\tau_{t h}=\overline{\theta^{2}} / 2 \varepsilon_{\theta \theta}$ or a hybrid one $\tau_{h}=\sqrt{\tau \tau_{t h}}$ should be more appropriate. However, because the heat-flux Equation 4 is linear in $\theta$, the use of $\tau_{t h}$ violates the linearity principle (Pope 1983).

The DNS results of Versteegh (1998) for a side-heated infinite vertical channel show that none of the coefficient is constant, even if nonlinear models are adopted, as in equation (22) below. Figure 4 illustrates the failure of the linear, quadratic, and cubic models of the slow pressure-scrambling term $\Pi_{\theta i, 1}$ to reproduce the

(a)

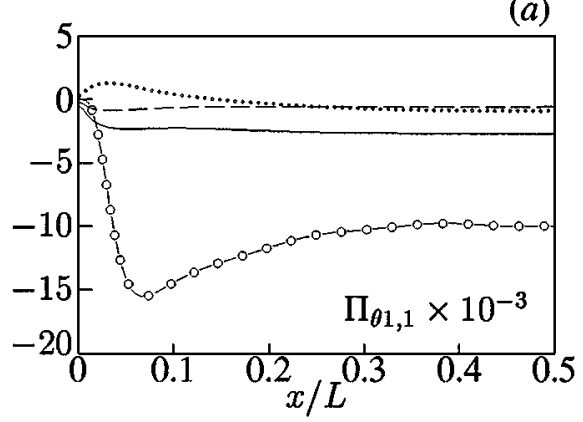

(b)

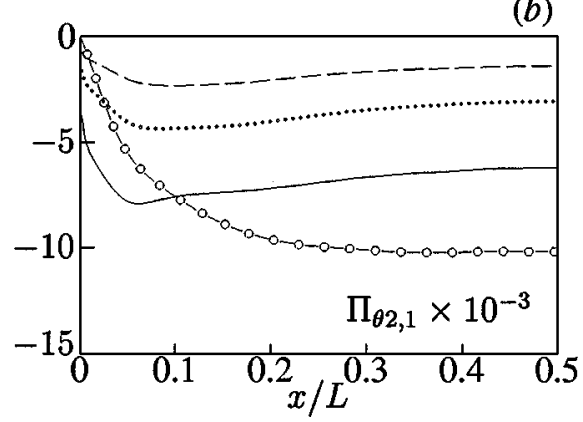

Figure 4 The slow part $\Pi_{\theta i, 1}(-\circ-)$ at $R a=5.4 \times 10^{5}$ for $i=1(a)$ and $i=2(b)$ compared with the variation of each, linear (-), quadratic $(\cdots)$, and cubic (--), term in Equation 22 for $C_{1 \theta}=C_{1 \theta}^{\prime}=C_{1 \theta}^{\prime \prime}=1$ (Dol et al. 1999). 
DNS results if constant coefficients are adopted (all coefficients are assigned a value of 1 for better illustration).

Dol et al. (1999) proposed an extension of the models for each part of $\Pi_{\theta i}$, which fits better with the DNS results for the considered case. The new models for the slow, rapid, and wall terms are

$$
\begin{gathered}
\Pi_{\theta i, 1}=-\frac{\varepsilon}{k}\left(C_{\theta 1} \overline{\theta u_{i}}+C_{\theta s}^{\prime} a_{i j} \overline{\theta u_{j}}+C_{\theta 1}^{\prime \prime} a_{i j} a_{i k} \overline{\theta u_{k}}\right), \\
\Pi_{\theta i, 2}=-C_{2 \theta} P_{\theta i}^{U}-C_{2 \theta}^{\prime} P_{\theta i}^{T}-C_{3 \theta} G_{\theta i},
\end{gathered}
$$

and

$$
\Pi_{\theta i}^{w}=C_{\theta}^{w}\left|a_{i j}\right|\left(\Pi_{\theta j, 1}+\Pi_{\theta j, 2}\right)
$$

where

$$
\begin{gathered}
C_{1 \theta}, C_{1 \theta}^{\prime}, C_{1 \theta}^{\prime \prime} \subset F_{p g r s}(A)=\frac{p[1-\exp (-q A)]}{1+r \exp (-s A)}, \\
C_{2 \theta}=1.25 A^{2} ; \quad C_{2 \theta}^{\prime}=6.15 A^{2}-19.3 A^{3}+15.0 A^{4} ; \quad C_{3 \theta}=0.45,
\end{gathered}
$$

and

$$
C_{\theta}^{w}=\max \left(0,0.58-0.69 A^{1 / 2}\right),
$$

where $a_{i j}=\overline{u_{i} u_{j}} / k-2 / 3 \delta_{i j}$ is the stress anisotropy tensor and $A=1-9 / 8\left(A_{2}-\right.$ $\left.A_{3}\right), A_{2}=a_{i j} a_{j i}$, and $A_{3}=a_{i j} a_{j k} a_{k i}$ are the stress anisotropy invariants. The values of the free coefficients $p, q, r$, and $s$ and more details about the model and its performance in a two-dimensional side-heated infinite channel can be found in Dol et al. (1999). Excellent reproduction of DNS results was achieved for all parts of $\Pi_{\theta i}$ and, most importantly, for the complete term for a range of Rayleigh numbers (see Figure 5).

The key to success is in the fine term-by-term tuning for a particular flow, but a successful application of the models to other flows is not guaranteed. Indeed, the above models applied to the same infinite plane channel but placed perpendicular to the gravitational vector with heating from below and cooling from above (Rayleigh-Bénard convection) do not perform satisfactorily, and additional tuning is needed. Recently, S. Gunarjo, S. Kenjereš, and K. Hanjalić (unpublished results) proposed additional modification aimed at Rayleigh-Bénard convection, while retaining the model form of Dol et al. (1999) (Equations 22 and 23). These modifications also lead to significant model simplification. Using the heat flux invariant $A_{\theta}=\left(\overline{\theta u_{i} \theta u_{i}} / k \overline{\theta^{2}}\right)^{1 / 2}$ instead of the stress invariant $A$, the new coefficients in the model of Dol et al. (1999) are

$$
C_{1 \theta}=\frac{p\left[1-\exp \left(-q A_{\theta}\right)\right]}{1+r \exp \left(-s A_{\theta}\right)}
$$


(a)

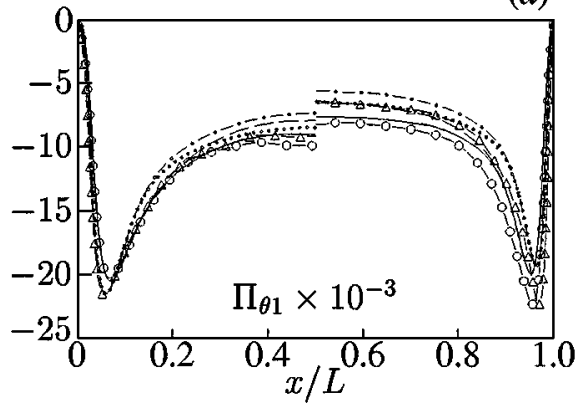

(b)

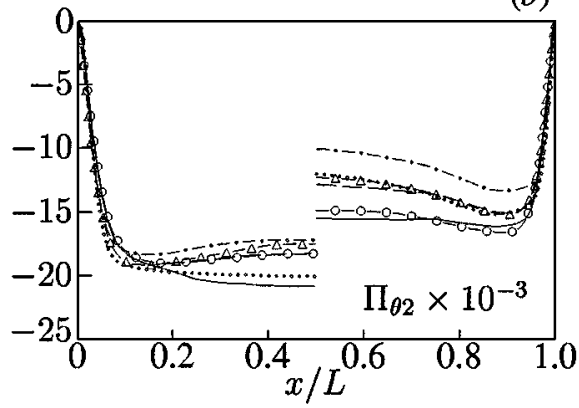

Figure 5 The new pressure-scrambling model (lines) compared with DNS (symbols) at several Rayleigh numbers. For $x / L<0.5$ : - and -o-, $R a=5.4 \times 10^{5} ; \cdots$ and $-\triangle-$, $R a=8.227 \times 10^{5} ; \mathrm{DNS}$. For $x / L>0.5$ : - and -०-, $R a=2 \times 10^{6} ; \cdots$ and $-\triangle-, R a=5 \times 10^{6}$ (Dol et al. 1999).

where $p=6, q=4, r=1, s=20$, and

$$
C_{1 \theta}^{\prime}=-2 C_{1 \theta}, \quad C_{2 \theta}=A_{\theta}, \quad C_{\theta}^{w}=0, \quad C_{2 \theta}^{\prime}=6 A_{\theta}^{2}-19 A_{\theta}^{3}+15 A_{\theta}^{4} .
$$

Admittedly, the use of $A_{\theta}$ is not consistent with the earlier mentioned linearity principle of Pope (1983). However, the model reproduces flux components both in vertical heated channel and in Rayleigh-Bénard convection, in reasonable agreement with the DNS results.

It should be recalled that in addition to Equation 4, one needs to model body force terms in the transport equation for turbulent stress tensor $\overline{u_{i} u_{j}}$ and in the scaleproviding equations, e.g., $\varepsilon$ and $\varepsilon_{\theta \theta}$, in which the task of deriving a general model is even more challenging. These problems, together with a large number of equations to be solved, have discouraged efforts toward development of a general secondmoment closure for flows driven by thermal and mass buoyancy. For practical application simpler models are used, tuned as a whole and not term-by-term, but with accepted compensating errors. An example is the algebraic flux model discussed next.

\subsection{Algebraic Flux Models (AFM)}

Second-moment closures can serve as a basis for deriving algebraic models, which do not require solutions of differential transport equations for each stress and flux component but can still capture important physical processes. By suitable elimination of differential (transport) terms, the differential equations for the second moments can be truncated to yield algebraic expressions for turbulent stress and heat and mass flux, e.g., Gibson \& Launder (1978) and Dol et al. (1997). Although such an approach for turbulent stresses leads to only limited success, algebraic truncation of the heat and mass flux seems more justified for buoyancy-dominated flows 
because of a strong coupling between the velocity and temperature/concentration fields through the buoyancy forces. The model of the slow term, either in linear or nonlinear form, is a crucial assumption for the truncation of Equation 4 because it enables the heat flux $\overline{\theta u_{i}}$ to be expressed explicitly. The following discussion should, in principle, be valid for any type of model for $\Pi_{\theta i}$ terms, provided that the model contains (among others) $\overline{\theta u_{i}}$ (Equations 21 and 22).

Depending on the level of truncation of Equation 4, different forms of algebraic models can be derived. The first truncation of Equation 4 can be performed by assuming the weak equilibrium hypothesis, which implies that the turbulence moments evolve more slowly than the imposed mean flow parameters and that the total transport (convection plus diffusion) of turbulence properties remains correlated. In the case of heat transport, this reduces to the assumption that the time evolution and the total transport of the correlation coefficient involving turbulent heat flux, i.e., $\overline{\theta u_{i}} / \sqrt{k} \sqrt{\theta^{2}}$, remain zero, i.e., $(\mathrm{D} / \mathrm{D} t-\mathcal{D})\left(\overline{\theta u_{i}} / \sqrt{k} \sqrt{\theta^{2}}\right)=0$, where $\mathrm{D} / \mathrm{D} t$ is material derivative and $\mathcal{D}$ is diffusive transport. Using the equations for turbulent kinetic energy and temperature variance, the weak equilibrium hypothesis leads to the truncation of Equation 4 into the following algebraic expression for turbulent heat flux:

$$
\overline{\theta u_{i}}=\frac{\overline{u_{i} u_{j}} \frac{\partial T}{\partial x_{j}}+\xi \overline{\theta u_{j}} \frac{\partial U_{i}}{\partial x_{j}}+\eta \beta g_{i} \overline{\theta^{2}}+\varepsilon_{\theta i}}{-C_{\theta 1} \frac{\varepsilon}{k}+\frac{1}{\overline{\theta^{2}}}\left(\overline{\theta u_{j}} \frac{\partial T}{\partial x_{j}}+\varepsilon_{\theta}\right)+\frac{1}{2 k}\left(\overline{u_{j} u_{k}} \frac{\partial U_{j}}{\partial x_{k}}+\beta g_{j} \overline{\theta u_{j}}+\varepsilon\right)} .
$$

This equation was derived by applying the first-order approximation for unknown correlations: gradient expression for turbulent diffusion, linear models for pressurescrambling terms $\Pi_{\theta i}$ (21) with coefficients $C_{\theta 1} \approx 3.0, C_{\theta 2}=C_{\theta 3} \approx 0.55$, yielding $\xi=1-C_{\theta 2}$ and $\eta=1-C_{\theta 3}$ both equal 0.45 .

DNS results can be used to validate the hypothesis of weak equilibrium on which the algebraic truncation is based (Dol et al. 1997, Kenjereš 1999). Figure $6 a$ shows a direct validation of the differential-to-algebraic truncation: In an infinite vertical channel the convection is zero so that the weak equilibrium expression reduces to the relation between the diffusion terms only, i.e., $2 \mathcal{D}_{\theta i}=\mathcal{D}_{k} \overline{\theta u_{i}} / k+\mathcal{D}_{\theta \theta} \overline{\theta u_{i}} / \overline{\theta^{2}}$. Departure from the above hypothesis in the near-wall regions is clearly shown. Figure $6 b$ provides an indirect test for Rayleigh-Bénard convection, showing that the correlation $\overline{\theta u_{i}} /\left(\overline{\theta^{2}} k\right)^{1 / 2}$ (as well as the timescale ratio) are not constant across the flow.

However, despite this failure to satisfy the DNS scrutiny, Equation 30 performs reasonably well in several relatively simple flows. Most probably, the expression involves some compensation of errors, which relax the above deficiency.

The same approach can be followed by adopting more advanced models of various source terms in the flux equation. For example, S. Gunarjo, S. Kenjereš, and K. Hanjalić (unpublished results) performed a priori validation of the algebraic model that contains their new model of $\Pi_{\theta i}$ (discussed above) (Equations 22, 

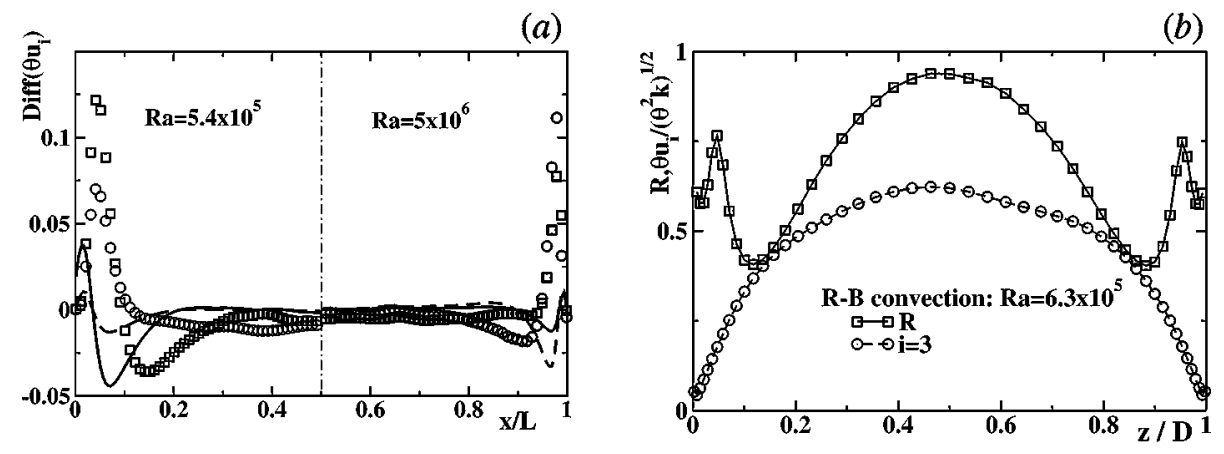

Figure 6 Test of differential-to-algebraic truncation: (a) diffusion flux in a vertical infinite side-heated channel and $(b)$ turbulent heat flux correlation from DNS in Rayleigh-Bénard convection. Also shown is the timescale ratio $R$. (Kenjereš 1999).

23, 28, and 29) and the Dol et al. (1999) model of flux dissipation. Figure 7 presents results of a priori computations of wall-normal heat flux for vertical $(a)$ and horizontal $(b)$ channels in close agreement with the DNS results. The model that reproduces well two generic cases, one with heating from the sides and one from below, has a good chance of performing in more general situations.

We note that Expression 30 has an implicit character and is nonlinear in $\overline{\theta u_{i}}$, with possibly multiple roots. When applied to complex flows, the expression may cause numerical difficulties (and even a singularity) if the denominator becomes too small in some flow regions. A possible remedy is to use the tensorial representation theorem to obtain an explicit expression (Girimaji \& Hanjalić 2000). Although such an approach offers a cure for possible numerical problems, its success in reproducing flow phenomena depends much on the original model of the parent differential Equation 4, primarily on the adopted model of term $\Pi_{\theta i}$.

(a)
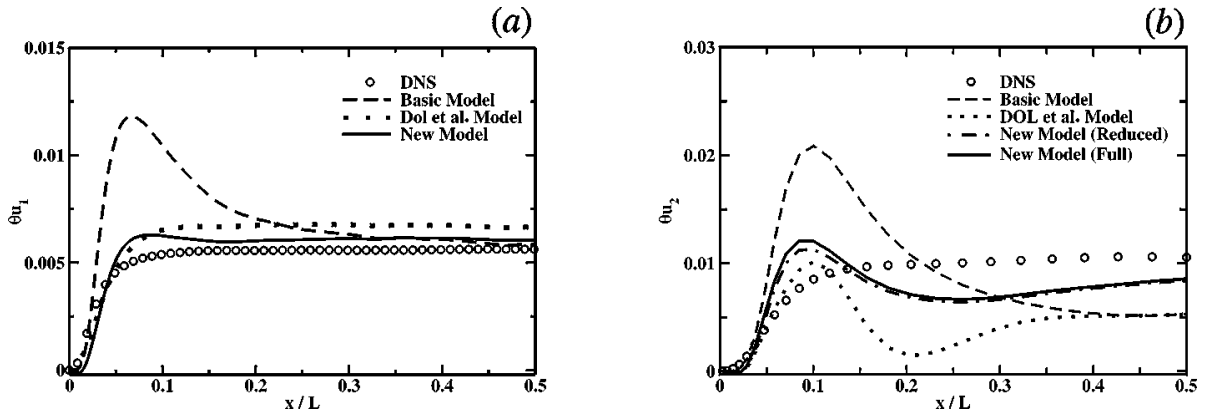

Figure 7 A priori validation of a new model for heat flux $(a)$ in a vertical infinite sideheated channel and $(b)$ in a Rayleigh-Bénard convection (DNS data used for all variables in the AFM expression) (Gunarjo et al. unpublished). 
A further simplification can be introduced by fully neglecting the transport terms in Equation 4, i.e., by assuming that $(\mathrm{D} / \mathrm{D}-\mathcal{D}) \overline{\theta u_{i}}=0$ (which essentially means that the production and dissipation of both $k$ and $\overline{\theta^{2}}$ are locally in balance), yielding the "reduced" algebraic expression

$$
\overline{\theta u_{i}}=-C^{\theta} \frac{k}{\varepsilon}\left(\overline{u_{i} u_{j}} \frac{\partial T}{\partial x_{j}}+\xi \overline{\theta u_{j}} \frac{\partial U_{i}}{\partial x_{j}}+\eta \beta g_{i} \overline{\theta^{2}}+\varepsilon_{\theta i}\right) .
$$

Although substantially simplified, Equation 31 still reflects the basic physics because it retains all three production terms from differential Equation 4, representing physical mechanisms that generate the turbulent heat flux. It is noted that neglecting the last two production terms (and $\varepsilon_{\theta i}$ ) leads to the nonisotropic eddydiffusivity model (also known as the generalized gradient diffusion hypothesis), $\overline{\theta u_{i}}=-C^{\theta}\left(k \overline{u_{i} u_{j}} / \varepsilon\right) \partial T / \partial x_{j}$, which can be regarded as a further step in the hierarchy of the truncation of Equation 4. Finally, replacing the turbulent stress $\overline{u_{i} u_{j}}$ by its trace $\overline{u_{i} u_{i}}=2 k$ leads to the isotropic eddy-diffusivity hypothesis (Equation 3 ). An analogous model hierarchy can be obtained for the turbulent stress $\overline{u_{i} u_{j}}$ and for the turbulent flux of species $\overline{s u_{i}}$. For example, the principle of retaining all source terms in algebraic truncation leads in the limit of local energy equilibrium to an extension of the conventional eddy-viscosity model for buoyancy-driven flows (with $\mathrm{C}$ to be determined):

$$
\overline{u_{i} u_{j}}=\frac{2}{3} k \delta_{i j}-v_{t}\left(\frac{\partial U_{i}}{\partial x_{j}}+\frac{\partial U_{j}}{\partial x_{i}}\right)+C \frac{k}{\varepsilon} \beta\left(g_{i} \overline{\theta u_{j}}+g_{j} \overline{\theta U_{i}}\right)
$$

The closure of the algebraic expressions, irrespective of the modeling level, requires that the basic scalar variables, $k, \overline{\theta^{2}}, \varepsilon$, and $\varepsilon_{\theta}$, be supplied from separate modeled transport equations. The resulting four-equation $k-\theta^{2}-\varepsilon-\varepsilon_{\theta}$ model is discussed in Hanjalić (1994). A further simplification can be achieved by expressing $\varepsilon_{\theta \theta}$ in terms of $\varepsilon, k$, and $\overline{\theta^{2}}$ from the assumed ratio of the thermal to mechanical turbulence timescales, $R=\tau_{\theta} / \tau$, with $R=$ const or prescribed by an algebraic function in terms of available variables. This reduces the model to a three-equation one, $k-\varepsilon-\overline{\theta^{2}}$. Although in many situations $R \neq c o n s t$, as shown above, such an assumption with the three-equation models displayed remarkable success in a number of flows (see next section).

\section{SOME ILLUSTRATIONS OF RANS-AFM APPLICATION}

Applications of the full differential second-moment closure model to the computation of buoyancy-driven flows are scarce in the literature and confined mainly to simple two-dimensional situations. Peeters \& Henkes (1992) reported computations of natural convection in a side-heated rectangular cavity using the basic model with some modifications of the dissipation $\varepsilon_{\theta i}$. Dol \& Hanjalić (2001) considered the same case but performed three-dimensional computations (nearcubic cavity) with several sets of boundary conditions (including nonisothermal 
horizontal walls) to match the experimental boundary conditions using a similar model. Dol et al. (1999) applied the modifications of the second-moment closure (discussed in Section 2.2) to predict the two-dimensional vertical channel with differentially heated walls. Although in all cases reported satisfactory agreement was achieved with available experimental and DNS data, the computations appeared to be lengthy and tedious especially for three-dimensional cases (17 differential equations and very fine numerical mesh in the near-wall regions), discouraging further testing in other more complex flows.

Algebraic models, based on rational truncation of the parent differential models, appeared to be more cost effective. Despite failing the DNS-based scrutiny of the founding weak equilibrium hypothesis, the reduced algebraic flux model (AFM) (Equation 31$)$ in the three-equation $\left(k-\theta^{2}-\varepsilon\right)$ or four-equation $\left(k-\overline{\theta^{2}}-\varepsilon-\varepsilon_{\theta \theta}\right)$ versions, tuned as a whole, produced satisfactory solutions in a range of enclosed and semienclosed buoyancy-driven turbulent flows with different geometries and boundary conditions (Hanjalić 1994, Kenjereš 1999). Four prerequisites seem to be crucial for this success: The algebraic flux expression (Equation 31) should contain all source terms, the full transport equation for $\overline{\theta^{2}}$ should be solved, equations need to be integrated up to the wall, and the flow needs to be computed at least in two dimensions, even if only one direction is inhomogeneous, such as in RayleighBénard convection. The following examples provide some illustrations.

\subsection{Horizontal Annuli with Heated Inner Cylinder}

At high $R a$ numbers, natural convection in horizontal annuli shows a stable longterm averaged flow pattern, characterized by a narrow turbulent thermal plume rising from the inner heated cylinder (Figure 8). After impinging on the top of the outer cylinder, the plume bifurcates into downward boundary layers along the interior walls of the outer cylinder, penetrating stably stratified side regions, eventually laminarizes and separates from the wall, and then turns upward and gets entrained by the central hot plume. This creates symmetric circulating motions
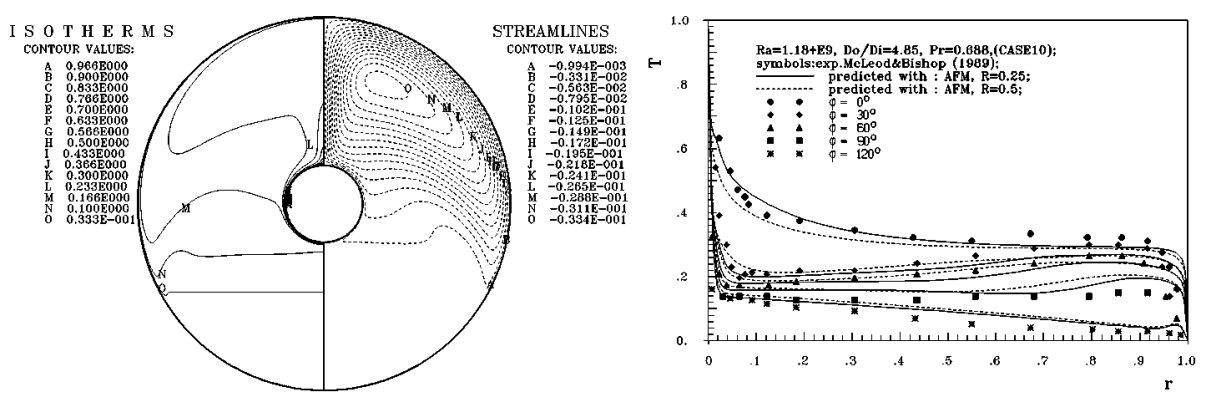

Figure 8 Two-dimensional RANS (AFM) of natural convection in a horizontal annulus with heated inner cylinder, compared with experiments, $D_{0} / D_{i}=4.85, R a=1,18 \times 10^{9}$, $\operatorname{Pr}=0.71$ (Kenjereš \& Hanjalić 1995). 
on both sides of the hot plume. Turbulence remains confined in the plume and wall boundary layers - particularly on the inner cylinder, with some remnants in the recirculating regions. The lower part of the annulus remains stagnant. The coexistence of these regimes (fully turbulent, transitional, laminar, and stagnant regions) poses a challenge to RANS modeling. In addition, the mean temperature across the recirculating regions shows an S-shaped profile with a positive gradient toward the outer cylinder, indicating an apparent counter-gradient transport. The two-dimensional RANS computation with the reduced algebraic model reproduces this pattern, well in accord with experiments (Figure 8) (Kenjereš \& Hanjalić 1995).

\subsection{Double Diffusive Ponds}

Another interesting challenge for RANS are double-diffusive systems, where the thermal and mass buoyancy counteract. An example of a bottom mixed layer, topped by a nonmixed stably stratified layer, acting as a conductive barrier, is shown in Figure $9 a$. Initially the salt-water solution is isothermal, with a linear, stable, mass stratification. Figure $9 b$ shows the time evolution of the mean temperature profiles, after the onset of bottom heating (Hanjalić \& Musemić 1997). The algebraic RANS model reproduced the uniform temperature and salt concentration profiles in the mixed layer, in accord with experiments.

\subsection{Two-Dimensional Computations of Finite-Length Rayleigh-Bénard Convection}

No model is capable of reproducing accurately the long-term averaged temperature profile in Rayleigh-Bénard convection, if treated as a one-dimensional problem with only the vertical coordinate as the independent variable. However, the two-dimensional computations with the RANS AFM model (Kenjereš \& Hanjalić
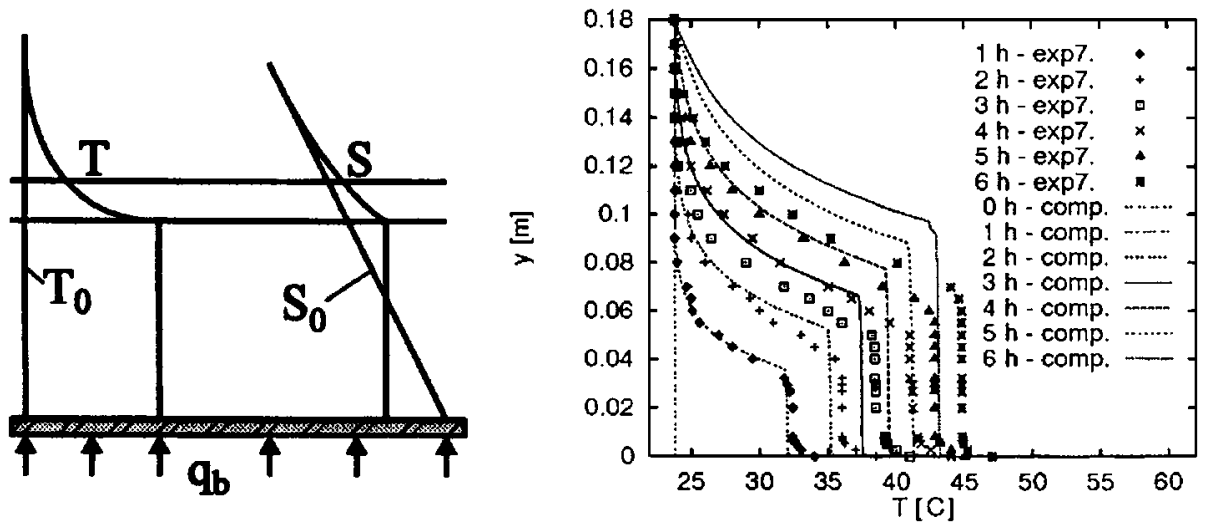

Figure 9 Schematic of temperature and concentration distribution in a double diffusive pond (left) and two-dimensional RANS computations of the time evolution of the mixed layer temperature, compared with experiments (Hanjalić \& Musemić 1997). 
2000) can reproduce not only the integral and local Nusselt numbers, but also the ensemble-averaged roll cell pattern (Figure 10). It is interesting to note that the computations for a 1:8 aspect ratio slender enclosure (and also for other cases with lower aspect ratios, not shown here) showed a step change in roll cell patterns and their wave lengths at $R a$ between $10^{8}$ and $10^{9}$.

\section{TRANSIENT RANS FOR VERY LARGE EDDY SIMULATION}

A way to capture the coherent large-scale structures, while still remaining within the RANS framework, is to combine the LES and RANS strategies. We presume that the flow variables can be decomposed into the ensemble-averaged part corresponding to very large coherent eddy structures and the incoherent part. The coherent part is then fully resolved by time integration of the threedimensional ensemble-averaged Navier-Stokes equations (having the same form as the Reynolds-averaged equations). The remaining incoherent part is modeled by a RANS-type closure, serving as the subscale model. Unlike in LES, the modeled part covers a significant portion of the turbulence spectrum, which means the cut-off filter is implicitly assumed to be at a much lower wave number than in traditional LES - (hence the acronym VLES). Consequently, both contributions to the turbulent fluctuations and long-term statistical averages are of an equal order of magnitude.

Modeling the larger part of the spectrum requires a more sophisticated model than the standard LES subgrid-scale model, which should not be related to the computational grid. Furthermore, because in the near-wall regions the RANS model provides a major contribution to turbulence statistics, it needs to account accurately for the wall phenomena. But, because the large-scale eddies are resolved, the demands are less strict than in traditional RANS where the full spectrum needs to be modeled. For example, resolving the large-scale motion enables one to capture accurately the large-scale convective transport, so that the subscale model can be of a simple algebraic type, dispensing with the need to solve full differential transport equations for second moments. Resolving the large-scale structure, which acts as the major carrier of momentum heat and species and also transports the turbulence, eliminates the problem of accurately modeling triple moments and pressure diffusion.

The solution of the resolved part of the spectrum can, in principle, follow the traditional LES practice using grid size as a basis for defining the filter or preferentially, as done in T-RANS, to solve ensemble- or conditionally averaged equations, which implicitly involve time filtering.

\subsection{The Time-Dependent RANS (T-RANS)}

The VLES approach targets complex technological and environmental flows with coherent structures at very high Reynolds and Rayleigh numbers where DNS 
VELOCITY VECTORS
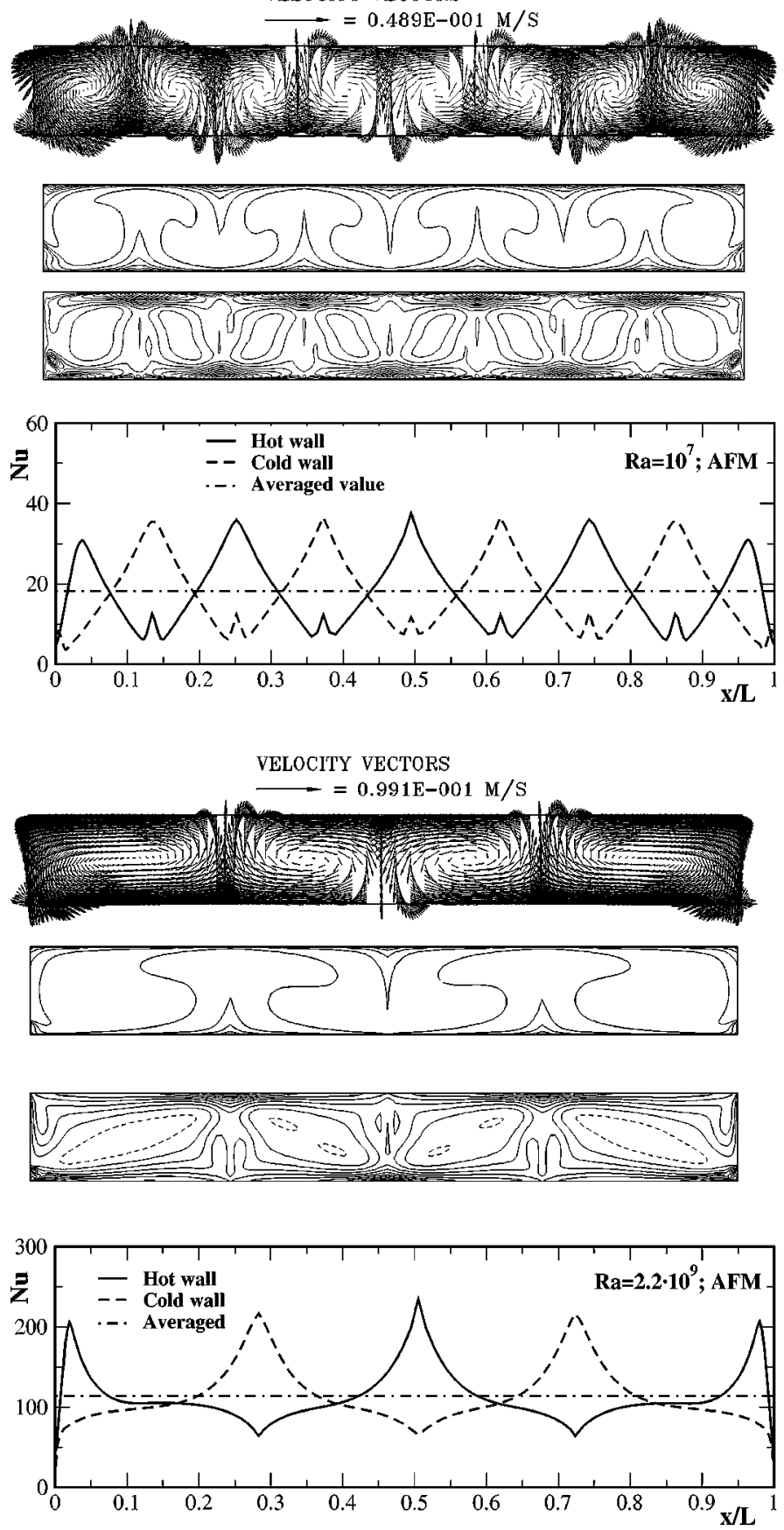
and LES are inapplicable. This is possible because one can use a coarser spatial grid than in LES. Close to solid walls, the grid needs to be fine but only in the wall-normal direction. Away from the wall, the grid can be coarser because the subscale model, here RANS, is less dependent on the spatial grid. The time step can be larger, allowing implicit time marching. If the resolved large-scale structure has a deterministic character, a relatively small number of realizations is needed to perform the averaging and to evaluate the turbulence statistics. The problem of defining inflow conditions at open boundaries is also less restrictive than in LES.

We consider here the classic Rayleigh-Bénard convection in which a distinct organized large-scale structure exists. The approach should also be applicable to other flows with a dominant large eddy structure (vortex shedding, internal separation and recirculation, longitudinal vortices, natural convection in enclosures, flows with rotation). In addition to accurately predicting flow features and heat transfer, we also demonstrate that the T-RANS can serve to identify the organized motion and its response to and reorganization due to imposed flow control methods, be it of a distributed type (extra body force), or a boundary control (nonplane wall configuration).

As illustrated above, T-RANS, though ensemble-averaged in the homogeneous direction, can also be used for two-dimensional simulations of flows with one homogeneous direction to reproduce a coherent large-scale structure, which is still more than a standard RANS can do (Figure 10) (Kenjereš \& Hanjalić 2000).

EQUATIONS FOR THE RESOLVED MOTION For incompressible fluid, the resolved motion is described by the ensemble-averaged momentum, continuity, and energy equation:

$$
\begin{aligned}
\frac{\partial\left\langle U_{i}\right\rangle}{\partial t}+\left\langle U_{j}\right\rangle \frac{\partial\left\langle U_{i}\right\rangle}{\partial x_{j}}= & \frac{\partial}{\partial x_{j}}\left(v \frac{\partial\left\langle U_{i}\right\rangle}{\partial x_{j}}-\tau_{i j}\right) \\
& -\frac{1}{\rho} \frac{\partial\left(\langle P\rangle-P_{r e f}\right)}{\partial x_{i}}+\beta g_{i}\left(\langle T\rangle-T_{r e f}\right) \\
\frac{\partial\langle T\rangle}{\partial t}+\left\langle U_{j}\right\rangle \frac{\partial\langle T\rangle}{\partial x_{j}} & =\frac{\partial}{\partial x_{j}}\left(\frac{v}{P r} \frac{\partial\langle T\rangle}{\partial x_{j}}-\tau_{\theta j}\right)
\end{aligned}
$$

where the \langle\rangle stands for resolved (implicitly filtered) quantities and $\tau_{i j}$ and $\tau_{\theta j}$ represent contributions due to unresolved scales to the momentum and temperature equation, respectively, provided by the subscale model. The buoyancy effects are assumed to comply with the Boussinesq approximation.

Figure 10 Two-dimensional T-RANS (AFM) solutions of natural convection in an enclosure (1:8 aspect ratio) heated from below. Velocity vectors and contours of mean temperature and vertical $(\overline{\theta v})$ turbulent heat flux. Top, $R a=10^{7}$ bottom, $R a=2.2 \times 10^{9}$ (Kenjereš \& Hanjalić 2000). 
MODEL FOR THE UNRESOLVED TURBULENCE Because only small-scale incoherent motion needs to be modeled, the reduced algebraic expression for heat flux $\tau_{\theta i}=\left\langle\theta u_{i}\right\rangle$ (Equation 31) proved earlier to reproduce well the wall thermal boundary layers in several generic situations, is selected to serve as the subscale model (note that all major flux production terms are retained but are treated as time dependent):

$$
\tau_{\theta i}=-C_{\theta} \frac{\langle k\rangle}{\langle\varepsilon\rangle}\left[\tau_{i j} \frac{\partial\langle T\rangle}{\partial x_{j}}+\xi \tau_{\theta j} \frac{\partial\left\langle U_{i}\right\rangle}{\partial x_{j}}+\eta \beta g_{i}\left\langle\theta^{2}\right\rangle\right],
$$

where $C_{\theta}=0.2, \xi=\eta=0.45$.

The turbulent stress tensor $\tau_{i j}=\left\langle u_{i} u_{j}\right\rangle$ should also be expressed in similar algebraic forms by truncation of the full transport equations. However, for Rayleigh-Bénard convection the simple eddy viscosity suffices because the largescale motions are fully resolved and the dominant turbulent stress component, which is the vertical one, is close to the turbulence kinetic energy.

The closure of the expressions for subscale quantities is achieved by solving in time the equations for turbulence kinetic energy $\langle k\rangle$, its dissipation rate $\langle\varepsilon\rangle$, and temperature variance $\left\langle\theta^{2}\right\rangle$ (all modified for low-Reynolds-number and near-wall effects), including the source buoyancy terms with standard coefficients and the thermal-to-mechanical turbulence timescale ratio $R=0.5$.

\subsection{Illustration of T-RANS Application}

The two-dimensional RANS-AFM can reproduce well the ensemble-averaged flow pattern and Nusselt numbers variation on the bounding walls in the classic Rayleigh-Bénard convection. However, if the flow geometry is more complex (inhomogeneous in all directions), if walls are not plane, or if an additional body force is applied, we need to perform full three-dimensional computations. When heating from below, the flow pattern is inherently unsteady, requiring timedependent treatment. To illustrate the potential of T-RANS we consider again the classic Rayleigh-Bénard convection. Figure 11 illustrates the capability of T-RANS to capture the instantaneous large convective structures at a very large range of Rayleigh numbers. To represent the structure morphology we consider planform structures with finger-like plumes in between (also detected by experiments). Figures $11 a$ and $11 b$ compare two instantaneous realizations at $R a=$ $6.5 \times 10^{5}$, one obtained by DNS (Wörner 1994) and the other by T-RANS. The resemblance is striking. The T-RANS cannot capture small-scale eddies, and these are missing in Figure $11 b$, but the overall picture looks very much the same as that obtained by DNS. Figure $11 c$ shows the same structure but for a much higher $R a$ number, $2 \times 10^{13}$. In accordance with two-dimensional RANS unsteady computations, Figure 10, the planform structures show much larger wave lengths, with a well-established boundary layer at the walls, which erupts into thin but strong plumes. At present only T-RANS is capable of yielding such morphological information at very high Rayleigh numbers. More details about the structure, as 
well as long-term averaged properties (mean temperature, second moments, wall heat transfer, etc.) can be found in Hanjalić \& Kenjereš $(1999,2000)$ and Kenjereš \& Hanjalić (1999a,b; 2000).

\section{CONCLUSIONS}

Potential and limitations of various closure levels of one-point turbulence models for flows driven or affected by thermal buoyancy are briefly reviewed. In the example of thermal convection in two generic flows, the side-heated vertical channel and Rayleigh-Bénard convection, it is demonstrated that the conventional isotropic eddy-diffusivity model with a constant turbulent Prandtl number is fundamentally wrong. Whereas second-moment closures are devoid of this and other deficiencies, a DNS-based term-by-term scrutiny of various terms in the basic second-moment closure reveals large discrepancies. Possible modifications of the second-moment closure to fit DNS results are discussed. Their a priori validation in some generic flows shows that it is possible to get very good term-by-term reproduction of DNS results, but their generality still remains questionable. Even if most of the terms can be modeled to reproduce DNS results in some generic situations, their inherent nonlinearity questions their generalization to complex flows. The DNS for some of the terms, e.g., in the $\varepsilon_{\theta \theta}$ equation, are still not available and are inaccessible by experiment.

However, even if most of the terms are modeled correctly, a full differential second-moment closure requires a substantial computational effort (solution of 17 partial differential equations for three-dimensional flows driven by thermal buoyancy, 24 equations for double diffusive systems, and still more if magnetic field, for example, is present).

A compromising closure level, using algebraic truncation of the second-moment closure, is shown to reproduce a number of internal flows driven by thermal and mass buoyancy. Despite failing a DNS scrutiny and inherent compensating errors, this level of modeling is recommended for industrial computations. The major prerequisites for success are retaining all source terms in the flux expressions, solving the transport equation for temperature variance in addition to kinetic energy and dissipation, and integrating equations up to the wall.

For three-dimensional flows with dominating large-scale eddy structures, no known one-point closure level is satisfactory. Fully resolving the large-scale eddies in time and space, while using the same above-mentioned algebraic RANS closure for modeling the remaining part of turbulence spectrum, can capture coherent structures and reproduce satisfactory all flow parameters and heat transfer. Illustration of such an approach, called T-RANS, is given in examples of Rayleigh-Bénard convection. Even two-dimensional simulations produce realistic ensemble-averaged patterns and spatial variation of wall Nusselt number. The real appeal of the T-RANS and similar types of very large eddy simulation is in their potential to handle very high Rayleigh numbers: Currently available simulations 
cover $R a$ numbers up to $10^{15}$, which are inaccessible to LES or any other available simulation techniques.

\section{ACKNOWLEDGMENTS}

The author thanks Dr. Saša Kenjereš and Dr. Henry Dol for fruitful discussion and for providing the computational results used for illustrations. Technical support of Erwin de Beus and Bart Hoek is also acknowledged.

\section{Visit the Annual Reviews home page at www.AnnualReviews.org}

\section{LITERATURE CITED}

Dol HS, Hanjalić K. 2001. Computational study of turbulent natural convection in a side-heated near-cubic enclosure at a high Rayleigh number. Int. J. Heat Mass Transf. 44:2323-44

Dol HS, Hanjalić K, Kenjereš S. 1997. A comparative assessment of the second-moment differential and algebraic models in turbulent natural convection. Int. J. Heat Fluid Flow 18:4-14

Dol HS, Hanjalić K, Versteegh TAM. 1999. A DNS-based thermal second-moment closure for buoyant convection at vertical walls. $J$. Fluid Mech. 391:211-47

Gibson MM, Launder BE. 1978. Ground effects on pressure fluctuations in the atmospheric boundary layer. J. Fluid Mech. 86: 491-511

Girimaji SS, Hanjalić K. 2000. Theoretical aspects of algebraic modeling of turbulent thermal flux. In Turbulence, Heat and Mass Transfer 3, ed. Y Nagano, K Hanjalić, T Tsuji, pp. 415-22. Tokyo, Jpn.: Aichi Shuppan

Grötzbach G. 1982. Direct numerical simulation of laminar and turbulent Bénard convection. J. Fluid Mech. 119:27-53

Hanjalić K. 1994. Achievements and limitations in modelling and computation of buoyant turbulent flows and heat transfer. In Proc. 10th Int. Heat Transf. Conf., ed. G Hewitt, pp. 1-18. London: IChem ${ }^{\mathrm{E}}$ /Taylor Francis

Hanjalić K, Jakirlić S, Hadžić I. 1997. Expanding the limits of equilibrium second-mo- ment turbulence closures. Fluid Dyn. Res. 20: 25-41

Hanjalić K, Kenjereš S. 2000a. Reorganization of turbulence structure in magnetic RayleighBénard convection: a T-RANS study. J. Turbul. 1(8):1-22

Hanjalić K, Kenjereš S. 2000b. T-RANS simulation of deterministic eddy structure and its effect on heat transfer in flows driven by thermal buoyancy and magnetic field. In Proc. 3rd Eur. Therm. Sci. Conf., ed. EWP Hahne, D Gorenflo, K Stephan, pp. 41-50. Pisa, Italy: Ed. ETS

Hanjalić K, Kenjereš S, Durst F. 1996. Natural convection in partitioned two-dimensional enclosures at high Rayleigh numbers. Int. J. Heat Mass Transf. 39:1407-27

Hanjalić K, Musemić R. 1997. Modelling the dynamics of double-diffusive scalar fields at various stability conditions. Int. J. Heat Fluid Flow 18:360-67

Kenjereš S. 1999. Numerical modelling of complex buoyancy-driven flows. $\mathrm{PhD}$ thesis. Delft Univ. Technol., Delft, The Netherlands

Kenjereš S, Hanjalić K. 1995. Prediction of turbulent thermal convection in concentric and eccentric annuli. Int. J. Heat Fluid Flow 16: 428-39

Kenjereš S, Hanjalić K. 1999a. Transient analysis of Rayleigh-Bénard convection with a RANS Model. Int. J. Heat Fluid Flow 20: 329-40

Kenjereš S, Hanjalić K. 1999b. Identification and visualization of coherent structures in 
Rayleigh-Bénard convection with a timedependent RANS. J. Vis. 2:169-76

Kenjereš S, Hanjalić K. 2000. Convective rolls and heat transfer in finite-length RayleighBénard convection: a two-dimensional study. Phys. Rev. E 62:7987-98

Kerr MR. 1996. Rayleigh number scaling in numerical convection. J. Fluid Mech. 310:13979

Launder BE. 1976. Heat and mass transport. In Turbulence, Topics in Applied Physics, ed. $\mathrm{P}$ Bradshaw, 12:177-86. Berlin: Springer

Lumley JL. 1978. Computational modeling of turbulent flows. Adv. Appl. Mech. 18:123-76

Peeters TWJ, Henkes RAWM. 1992. The Reynolds-stress model of turbulence applied to natural convection boundary layer along a heated vertical plate. Int. J. Heat Mass Transf. $33: 403-20$
Pope S. 1983. Consistent modeling of scalars in turbulent flows. Phys. Fluids 26:404-8

Shih T-H, Lumley JL, Chen J-Y. 1988. Secondorder modelling of a passive scalar in a turbulent shear flow. AIAA J. 28:610-17

Versteegh TAM. 1998. Numerical simulation of natural convection in a differentially heated vertical channel. $\mathrm{PhD}$ thesis. Delft Univ. Technol., Delft, The Netherlands

Wörner M. 1994. Direkte Simulation turbulenter Rayleigh-Bénard Konvektion in füssigem Natrium. $\mathrm{PhD}$ thesis. Univ. Karlsruhe, Germany

Wörner M, Grötzbach G. 1997. Pressure transport in DNS of turbulent natural convection in horizontal fluid layers. In Turbulence, Heat and Mass Transfer 2, ed. K Hanjalić, TWJ Peeters, pp. 351-60. Delft, The Netherlands: Delft Univ. Press 

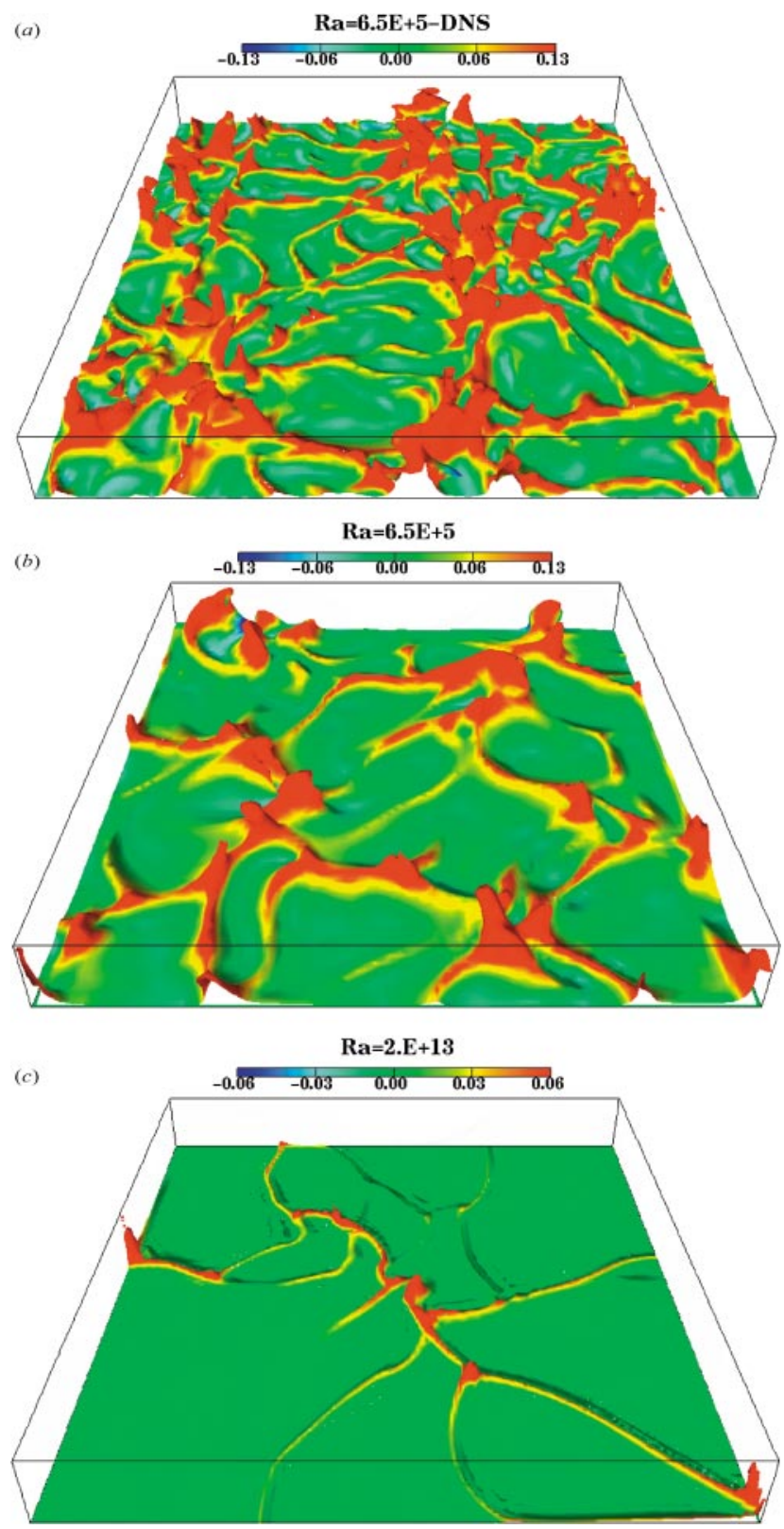

Figure 11 Planform structures with finger-like plumes in between (temperature isosurfaces are colored by the intensity of the vertical velocity): (a) DNS, $R a=6.5 \times 10^{5}$, (b) T-RANS, $R a=6.5 \times 10^{5}$, (c) T-RANS, $R a=2 \times 10^{13}$. (S. Kenjereš \& K. Hanjalić, unpublished data.) 


\section{CONTENTS}

FRONTISPIECE

Milton VAN DyKe, THE MAN AND hIS WORK, Leonard W. Schwartz 1

G.K. BATCHELOR AND THE HOMOGENIZATION OF TURBULENCE, H.K. Moffatt

DAVID CRIGHTON, 1942-2000: A COMMENTARY ON HIS CAREER AND HIS INFLUENCE ON AEROACOUSTIC THEORY, John E. Ffowcs Williams 37

Sound Propagation Close to the Ground, Keith Attenborough 51

ELLIPTICAL INSTABILITY, Richard R. Kerswell 83

LAgRANGIAN INVESTIGATIONS OF TURBULENCE, P.K. Yeung 115

CAVitation in Vortical Flows, Roger E.A. Arndt 143

Microstructural Evolution in Polymer Blends, Charles $L$. Tucker III and Paula Moldenaers 177

Cellular Fluid Mechanics, Roger D. Kamm 211

DynAmiCAl PHENOMENA In LIQUID-CRYSTALline MATERIALS, Alejandro D. Rey and Morton M. Denn 233

Noncoalescence and Nonwetting Behavior of LiQuids, G. Paul Neitzel and Pasquale Dell' Aversana

BOUNDARY-LAYER RECEPTIVITY TO FREESTREAM DISTURBANCES, William S. Saric, Helen L. Reed, and Edward J. Kerschen

One-Point Closure Models For BuOyancy-Driven Turbulent FLOWS, K. Hanjalić

Wall-Layer Models for Large-EdDy Simulations, Ugo Piomelli and Elias Balaras

FILAMENT-STRETCHING RHEOMETRY OF COMPLEX FLUIDS, Gareth $H$. McKinley and Tamarapu Sridhar

MoleCUlar ORIENTATION EFFECTS IN VisCOElasticity, Jason K.C. Suen, Yong Lak Joo, and Robert C. Armstrong

THE RICHTMYER-MESHKOV INSTABILITY, Martin Brouillette

SHIP WAKES AND THEIR RADAR IMAGES, Arthur M. Reed and Jerome H. Milgram 
SyNTHETIC JETS, Ari Glezer and Michael Amitay

FluID DYNAMICS OF El NiÑO VARIABILITY, Henk A. Dijkstra and

Gerrit Burgers

INTERNAL GRAVITY WAVES: FROM INSTABILITIES TO TURBULENCE,

C. Staquet and J. Sommeria

INDEXES

Subject Index

Cumulative Index of Contributing Authors, Volumes 1-34

Cumulative Index of Chapter Titles, Volumes 1-34

\section{ERRATA}

An online log of corrections to the Annual Review of Fluid Mechanics chapters may be found at http://fluid.annualreviews.org/errata.shtml 